\title{
CONJUGATE PRIORS FOR EXPONENTIAL-TYPE PROCESSES WITH RANDOM INITIAL CONDITIONS
}

Abstract. The family of proper conjugate priors is characterized in a general exponential model for stochastic processes which may start from a random state and/or time.

1. Introduction and preliminaries. The notion of conjugate priors plays a fundamental role in modern Bayesian statistics. Diaconis and Ylvisaker (1979) characterized proper priors in the exponential families $P_{\theta}$, $\theta \in \Theta$, of distributions determined by

$$
\frac{d P_{\theta}}{d \mu}=\exp \left[\sum_{i=1}^{n} \theta_{i} x_{i}-\Phi(\theta)\right]
$$

where $\mu$ is a $\sigma$-finite measure on $\mathbb{R}^{n}$. Let $\mathcal{X}$ be the interior of the convex hull of the support set of the measure $\mu$ and let $\Theta$ be a nonempty open set in $\mathbb{R}^{n}$. Let $\pi\left(\theta ; n_{0}, x_{0}\right), n_{0} \in \mathbb{R}, x_{0} \in \mathbb{R}^{n}$, be a prior on $\Theta$ defined by $d \pi\left(\theta ; n_{0}, x_{0}\right)=\exp \left[n_{0}\left(x_{0} \theta-\Phi(\theta)\right)\right] d \theta$. Assuming that $\mathcal{X}$ is a nonempty open set in $\mathbb{R}^{n}$, Diaconis and Ylvisaker (1979) proved the following two theorems:

Theorem 1. If $n_{0}>0$ and $x_{0} \in \mathcal{X}$, then $\pi\left(\Theta ; n_{0}, x_{0}\right)<\infty$. Conversely, if $\pi\left(\Theta ; n_{0}, x_{0}\right)<\infty$ and $\Theta=\mathbb{R}^{n}$, then $n_{0}>0$; while if $\pi\left(\Theta ; n_{0}, x_{0}\right)<\infty$ with $n_{0}>0$, then $x_{0} \in \mathcal{X}$.

ThEOREM 2. Suppose that $\Theta$ is open in $\mathbb{R}^{n}$. If $\theta$ has the distribution $\pi\left(\theta ; n_{0}, x_{0}\right), n_{0}>0$ and $x_{0} \in \mathcal{X}$, then the expected value of $\nabla \Phi(\theta)$ is $x_{0}$.

In this paper the following general exponential model for stochastic processes is considered. Let $X(t), t \in T$, be a stochastic process defined on a probability space $\left(\Omega, \mathcal{F}, P_{\theta}\right)$ with values in $\left(\mathbb{R}^{k}, \mathcal{B}_{\mathbb{R}^{k}}\right)$, where $T=[0, \infty)$ or

1991 Mathematics Subject Classification: Primary 62E10, 62F15.

Key words and phrases: conjugate prior, exponential-type process, stopping time. 
$T=\{0,1,2, \ldots\}$ and $\theta$ is a parameter with values in an open set $\Theta \subset \mathbb{R}^{n}$. Let $P_{\theta, t}$ denote the restriction of $P_{\theta}$ to the $\sigma$-algebra $\mathcal{F}_{t}=\sigma\{X(s): s \leq t\}$. Suppose that the family $P_{\theta, t}, \theta \in \Theta$, is dominated by a measure $\mu_{t}$ which is the restriction of a probability measure $\mu$ to $\mathcal{F}_{t}$. Moreover, assume that the density functions (likelihood functions) have the following exponential form:

$$
\frac{d P_{\theta, t}}{d \mu_{t}}=\exp \left[\sum_{i=1}^{n} \theta_{i} Z_{i}(t)-\Phi(\theta) S(t)-\Psi(\theta)\right],
$$

where both $\Phi(\theta)$ and $\Psi(\theta), \theta \in \Theta$, are real and strictly convex functions, and $(Z(t), S(t)), t \in T$, is a stochastic process adapted to the filtration $\mathcal{F}_{t}$, $t \in T$. Clearly, $(Z(t), S(t))$ is a sufficient statistic for $\theta$ relative to $\mathcal{F}_{t}, t \in T$.

The process $(Z(t), S(t)), t \in T$, is assumed to satisfy the following conditions: $Z(t)$ is right continuous as a function of $t, P_{\theta^{-}}$a.s., and $S(t), t \in T$, are nonnegative random variables $(S(t)$ may be nonrandom as well) such that $S(t)$ is strictly increasing and continuous as a function of $t$ and $S(t) \rightarrow \infty$ as $t \rightarrow \infty, P_{\theta}$-a.s.

Upon admissible interchanges of differentiation and integration, differentiating the identity $\int d P_{\theta, t}=1$ in $\theta$ yields

$$
\begin{aligned}
E_{\theta} Z(t) & =\nabla \Phi(\theta) E_{\theta} S(t)+\nabla \Psi(\theta) \\
& =\left(\frac{\partial \Phi(\theta)}{\partial \theta_{1}}, \ldots, \frac{\partial \Phi(\theta)}{\partial \theta_{n}}\right)^{*} E_{\theta} S(t)+\left(\frac{\partial \Psi(\theta)}{\partial \theta_{1}}, \ldots, \frac{\partial \Psi(\theta)}{\partial \theta_{n}}\right)^{*} \\
& =\left(\frac{\partial \Phi(\theta)}{\partial \theta_{1}} E_{\theta} S(t)+\frac{\partial \Psi(\theta)}{\partial \theta_{1}}, \ldots, \frac{\partial \Phi(\theta)}{\partial \theta_{n}} E_{\theta} S(t)+\frac{\partial \Psi(\theta)}{\partial \theta_{n}}\right)^{*}
\end{aligned}
$$

and

$$
\begin{aligned}
E_{\theta}[Z(t) & -\nabla \Phi(\theta) S(t)-\nabla \Psi(\theta)][Z(t)-\nabla \Phi(\theta) S(t)-\nabla \Psi(\theta)]^{*} \\
& =\Phi^{\prime \prime}(\theta) E_{\theta} S(t)+\Psi^{\prime \prime}(\theta)=\left(\frac{\partial^{2} \Phi(\theta)}{\partial \theta_{i} \partial \theta_{j}}\right)_{i, j=1}^{n} E_{\theta} S(t)+\left(\frac{\partial^{2} \Psi(\theta)}{\partial \theta_{i} \partial \theta_{j}}\right)_{i, j=1}^{n} \\
& =\left(\frac{\partial^{2} \Phi(\theta)}{\partial \theta_{i} \partial \theta_{j}} E_{\theta} S(t)+\frac{\partial^{2} \Psi(\theta)}{\partial \theta_{i} \partial \theta_{j}}\right)_{i, j=1}^{n} .
\end{aligned}
$$

Here the star denotes transposition.

This class of processes includes many counting, branching, diffusiontype etc. processes and the family of exponential-type processes which may start from a random state and/or time, comprising also some models for stationary Gaussian processes. In solving problems of Bayes and minimax estimation for this model there arises the question of characterizing the family of proper priors on $\Theta$ which should be conjugate to the family of (1). In the paper of Magiera and Wilczyński (1991) the model corresponding to (1) 
with $\Psi(\theta) \equiv 0, Z(0)=0$ and $S(0)=0$ was considered. The conjugate prior distributions associated with that model include most well-known distributions; in contrast, the general model considered in this paper supplies new families of priors.

In the terminology of Barndorff-Nielsen (1980), the exponential family given by $(1)$ is in general an $(n+1, n)$-curved exponential family $((n+1, n)$ c.e.f.), while that considered by Diaconis and Ylvisaker (1979) is a non-c.e.f. A sequential version of (1) also becomes an $(n+1, n)$-c.e.f. even in the case when $S(t) \equiv t$. Generalizing the results of Diaconis and Ylvisaker (1979), in this paper the family of proper priors on $\Theta$ which are conjugate to the c.e.f.'s defined by (1) is characterized. It is also shown that for this family of priors and for any stopping time $\tau$ with respect to $\mathcal{F}_{t}, t \in T$, the posterior expectations of $\nabla \Phi(\theta)$ and $\nabla \Psi(\theta)$ given $\mathcal{F}_{\tau}$ satisfy

$$
\frac{n_{0} s+S(\tau)}{n_{0}+1} E\left[\nabla \Phi(\theta) \mid \mathcal{F}_{\tau}\right]+E\left[\nabla \Psi(\theta) \mid \mathcal{F}_{\tau}\right]=\frac{n_{0} z+Z(\tau)}{n_{0}+1},
$$

where $n_{0}, z, s$ are hyperparameters of the prior distribution. Some special models of (1) are also considered. Examining properties of the new families of priors, one olso obtains some recurrent relations for parabolic cylinder functions.

2. Conjugate priors for exponential-type processes. The statistical model for stochastic processes, defined by (1), is essentially more general than that considered in Magiera and Wilczyński (1991) (it also contains some models for stationary Gaussian processes and for other processes starting from a random state and/or time) and it is much more general than the one corresponding to non-c.e.f's. Just as in Magiera and Wilczyński (1991), to take the full force of Diaconis and Ylvisaker's results which hold for the latter model, the idea of transforming the curved exponential family into a non-c.e.f. by using a stopping time will be applied.

Let $\tau$ be a Markov stopping time with respect to $\mathcal{F}_{t}, t \in T$, such that $P_{\theta}(\tau<\infty)=1$ for each $\theta \in \Theta$. Then, by a modification of a lemma of Sudakov (cf. Döhler (1981)), a sequential version of the likelihood function (1) is

$$
\frac{d P_{\theta, \tau}}{d \mu_{\tau}}=\exp \left[\sum_{i=1}^{n} \theta_{i} Z_{i}(\tau)-\Phi(\theta) S(\tau)-\Psi(\theta)\right],
$$

Clearly, the exponential family of $(2)$ is in general an $(n+1, n)$-c.e.f.

Denote by $\mathcal{S}$ the set of all possible values of the process $S(t), t \in T$. For each $s$ in $\mathcal{S}$ define the following stopping time:

$$
\tau_{s}=\inf \{t \in T: S(t)=s\}
$$


It follows from the assumptions imposed on $S(t)$ that

$$
P_{\theta}\left(\tau_{s}<\infty\right)=1, \quad \theta \in \Theta, s \in \mathcal{S},
$$

which, by (2), implies that

$$
\frac{d P_{\theta, \tau_{s}}}{d \mu_{\tau_{s}}}=\exp \left[\sum_{i=1}^{n} \theta_{i} Z_{i}\left(\tau_{s}\right)-\Phi(\theta) s-\Psi(\theta)\right] .
$$

Obviously, the likelihood function (3) is a non-c.e.f.

For each $s \in \mathcal{S}$, let

$$
Z_{s}=\operatorname{int}\left(\operatorname{conv} \operatorname{supp} \mu_{\tau_{s}}\right) .
$$

As in Diaconis and Ylvisaker (1979), it will be assumed that $Z_{s}$ is a nonempty open set in $\mathbb{R}^{n}$. Further, for all $s \in \mathcal{S}$, let

$$
\mathcal{X}_{s}=\left\{(z, s): z \in Z_{s}\right\} \quad \text { and } \quad \mathcal{X}=\operatorname{conv}\left(\bigcup_{s \in \mathcal{S}} \mathcal{X}_{s}\right) .
$$

Define a family $\pi\left(\theta ; n_{0}, z, s\right), n_{0} \in \mathbb{R},(z, s) \in \mathbb{R}^{n+1}$, of measures on $\Theta$ with density (with respect to the Lebesgue measure) given by

$$
f\left(\theta ; n_{0}, z, s\right)=\exp \left[n_{0}(z \theta-M(\theta ; s))\right],
$$

where $M(\theta ; s)=\Phi(\theta) s+\Psi(\theta)$. The following theorem generalizes the result of Diaconis and Ylvisaker (1979).

Theorem 3. If $n_{0}>0$ and $(z, s) \in \mathcal{X}$, then

$$
\begin{gathered}
\int_{\Theta} f\left(\theta ; n_{0}, z, s\right) d \theta<\infty, \\
\int_{\Theta}(\nabla M(\theta ; s)) f\left(\theta ; n_{0}, z, s\right) d \theta=z .
\end{gathered}
$$

Taking into account the facts given above, this theorem can be proven by the methods used by Diaconis and Ylvisaker (1979) in their proofs of Theorems 1 and 2 .

Define $M_{i}(\theta ; s)=\left(\partial / \partial \theta_{i}\right) M(\theta ; s)$ and $M_{i j}(\theta ; s)=\left(\partial^{2} / \partial \theta_{i} \partial \theta_{j}\right) M(\theta ; s)$, $i, j=1, \ldots, n$. The following lemma can be useful in deriving posterior risks when a quadratic loss function is used.

Lemma. Suppose that $\operatorname{EM}_{i j}(\theta ; s)<\infty$ and

$$
\int_{\Theta} \frac{\partial}{\partial \theta_{j}}\left\{\left[z_{i}-M_{i}(\theta ; s)\right] \exp \left[n_{0}(z \theta-M(\theta ; s))\right]\right\} d \theta=0, \quad i, j=1, \ldots, n,
$$

for some $n_{0}>0$ and $(z, s) \in \mathcal{X}$. Then

$$
E[z-\nabla M(\theta ; s)][z-\nabla M(\theta ; s)]^{*}=\frac{1}{n_{0}} E M^{\prime \prime}(\theta ; s) .
$$


Proof. Note that

$$
\begin{aligned}
\nabla f\left(\theta ; n_{0}, z, s\right)= & n_{0}[z-\nabla M(\theta ; s)] f\left(\theta ; n_{0}, z, s\right), \\
f^{\prime \prime}\left(\theta ; n_{0}, z, s\right)= & \left\{n_{0}^{2}[z-\nabla M(\theta ; s)][z-\nabla M(\theta ; s)]^{*}\right. \\
& \left.-n_{0} M^{\prime \prime}(\theta ; s)\right\} f\left(\theta ; n_{0}, z, s\right) .
\end{aligned}
$$

In view of the assumptions of the lemma, integrating the latter equality over $\Theta$ yields formula (5).

Let $\left(X^{(1)}(t), \ldots, X^{(N)}(t)\right)$ be a random sample of size $N$ from $P_{\theta}$. Define

$$
\bar{Z}(t)=\frac{1}{N} \sum_{i=1}^{N} Z^{(i)}(t), \quad \bar{S}(t)=\frac{1}{N} \sum_{i=1}^{N} S^{(i)}(t) .
$$

The following theorem is an immediate consequence of Theorem 3 and the sequential version of likelihood functions given by (2).

THEOREM 4. Let $\tau$ be any finite stopping time with respect to $\mathcal{F}_{t}=$ $\sigma\left\{X^{(1)}(s), \ldots, X^{(N)}(s): s \leq t\right\}, t \geq 0$. If $\pi\left(\theta ; n_{0}, z, s\right)$ is the prior distribution of $\theta$, then the posterior distribution of $\theta$ given $\mathcal{F}_{\tau}$ is $\pi\left(\theta ; n_{0}+N, r_{\tau}, \alpha_{\tau}\right)$, where

Moreover,

$$
r_{\tau}=\frac{n_{0} z+N \bar{Z}(\tau)}{n_{0}+N}, \quad \alpha_{\tau}=\frac{n_{0} s+N \bar{S}(\tau)}{n_{0}+N} .
$$

$$
E\left(\nabla M\left(\theta ; \alpha_{\tau}\right) \mid \mathcal{F}_{\tau}\right)=r_{\tau} .
$$

Let us note that all the above results remain true when $\mathcal{X}$ is replaced by the interior $\mathcal{Y}$ of the convex hull of the set of all possible values of the process $(Z(t), S(t)), t \in T$ (because $\mathcal{Y} \subseteq \mathcal{X}$ ).

3. Some special models. Consider now some particular cases of the general model considered. The models of Examples 1-3 below were considered by Magiera and Stefanov (1989) in efficient sequential estimation. In this section, considering these models in the context of Bayesian analysis, certain new families of priors will be exhibited. As a by-product, one obtains in Example 1 certain recurrent relations for parabolic cylinder functions.

EXAmple 1 (the Ornstein-Uhlenbeck velocity process). Let $X(t), t \in$ $T=[0, \infty)$, be a stochastic process satisfying the stochastic differential equation

$$
d X(t)=-\theta X(t) d t+d W(t),
$$

where $W(t), t \in T$, denotes the standard Wiener process and $X(0)$ is normally distributed with zero mean and variance $(2 \theta)^{-1}, \theta \in \Theta=(0, \infty)$. The process $X(t), t \in T$, is a stationary Gaussian Markov process with 
$E_{\theta} X(t)=0$ and covariance function $B(s, t)=(2 \theta)^{-1} \exp (-\theta|t-s|)$. The likelihood function for this process is

$$
\frac{d P_{\theta, t}}{d \mu_{t}}=\theta^{1 / 2} \exp \left\{-\frac{1}{2} \theta\left[X^{2}(0)+X^{2}(t)-t+\theta \int_{0}^{t} X^{2}(s) d s\right]\right\} .
$$

Thus the process belongs to the family defined by (1) with $Z(t)=\frac{1}{2}[t-$ $\left.X^{2}(t)-X^{2}(0)\right], S(t)=\int_{0}^{t} X^{2}(s) d s, \Phi(\theta)=\theta^{2} / 2$ and $\Psi(\theta)=-\frac{1}{2} \log \theta$.

The prior distribution $\pi\left(\theta ; n_{0}, z, s\right)$ of the parameter $\theta$ has the density

$$
f\left(\theta ; n_{0}, z, s\right)=K\left(n_{0}, z, s\right) \theta^{n_{0} / 2} \exp \left[n_{0}\left(z \theta-s \frac{\theta^{2}}{2}\right)\right],
$$

$n_{0}>0,(z, s) \in \mathcal{Y}=(-\infty, \infty) \times(0, \infty)$. By using formula 3.462 .1 of Gradshteŭn and Ryzhik (1971) the norming constant $K\left(n_{0}, z, s\right)$ can be expressed by

$$
\begin{aligned}
& {\left[K\left(n_{0}, z, s\right)\right]^{-1}} \\
& \quad=\left(n_{0} s\right)^{-\left(n_{0}+2\right) / 4} \Gamma\left(\frac{n_{0}+2}{2}\right) \exp \left(\frac{n_{0} z^{2}}{4 s}\right) \mathcal{D}_{-\left(n_{0}+2\right) / 2}\left(-\left(\frac{n_{0}}{s}\right)^{1 / 2} z\right),
\end{aligned}
$$

where $\mathcal{D}_{\nu}(\kappa)$ denotes the parabolic cylinder function

$$
\mathcal{D}_{\nu}(\kappa)=\frac{\exp \left(-\kappa^{2} / 4\right)}{\Gamma(-\nu)} \int_{0}^{\infty} \theta^{-\nu-1} \exp \left(-\kappa \theta-\theta^{2} / 2\right) d \theta, \quad \nu<0 .
$$

In particular, for $z=0$,

$$
\left[K\left(n_{0}, 0, s\right)\right]^{-1}=\frac{1}{2}\left(\frac{2}{n_{0} s}\right)^{\left(n_{0}+2\right) / 4} \Gamma\left(\frac{n_{0}+2}{4}\right) .
$$

By Theorem 3(ii),

$$
E\left(s \theta-\frac{1}{2 \theta}\right)=z .
$$

For the process considered, the conditions of the Lemma are satisfied for all $n_{0}>2$ and $(z, s) \in \mathcal{Y}$. Thus, by (5),

$$
D\left[s \theta-(2 \theta)^{-1}\right]=E\left[z-s \theta+(2 \theta)^{-1}\right]^{2}=\frac{1}{n_{0}}\left(s+\frac{1}{2} E \theta^{-2}\right),
$$

or equivalently,

(6) $4 n_{0} s^{2} E \theta^{2}+\left(n_{0}-2\right) E \theta^{-2}=4\left[n_{0}\left(z^{2}+s\right)+s\right]$,

$n_{0}>2,(z, s) \in \mathcal{Y}$. Equality (6) leads to the following relation:

$$
\begin{aligned}
\left(n_{0}+2\right)\left(n_{0}+4\right) s \mathcal{D}_{-n_{0} / 2-3}(\kappa)+4 s \mathcal{D}_{-n_{0} / 2+1}(\kappa) & \\
& =4\left[n_{0}\left(z^{2}+s\right)+s\right] \mathcal{D}_{-n_{0} / 2-1}(\kappa),
\end{aligned}
$$


$n_{0}>2, s>0$, where $\kappa$ is related to $n_{0}$ through $\kappa=-\left(n_{0} / s\right)^{1 / 2} z$. Putting $p=-\left(n_{0}+2\right) / 2$ yields the following recurrent formula for parabolic cylinder functions:

$$
\mathcal{D}_{p+2}(\kappa)+\left(2 p-\kappa^{2}+1\right) \mathcal{D}_{p}(\kappa)+p(p-1) \mathcal{D}_{p-2}(\kappa)=0,
$$

$p<-2$.

ExAmple 2. Consider the two-dimensional stationary zero-mean Gaussian Markov process $X(t)=\left(X_{1}(t), X_{2}(t)\right), t \in T=[0, \infty)$, satisfying the following system of stochastic differential equations:

$$
\begin{aligned}
& d X_{1}(t)=-\left[\theta_{1} X_{1}(t)+\theta_{2} X_{2}(t)\right] d t+d W_{1}(t), \\
& d X_{2}(t)=\left[\theta_{2} X_{1}(t)-\theta_{1} X_{2}(t)\right] d t+d W_{2}(t),
\end{aligned}
$$

where $\left(W_{1}(t), W_{2}(t)\right), t \in T$, is a Wiener process with independent components and independent of $X(0)$, and $\theta_{1}, \theta_{2}$ are parameters such that $\theta_{1}>0$, $-\infty<\theta_{2}<\infty$.

By Lemma 17.5 of Liptser and Shiryaev (1978), for every $t \in T$ the components of the vector $\left(X_{1}(t), X_{2}(t)\right)$ are independent and have identical variances equal to $\left(2 \theta_{1}\right)^{-1}$. Thus the vector $\left(X_{1}(0), X_{2}(0)\right)$ has density $\left(\theta_{1} / \pi\right) \exp \left[-\theta_{1}\left(x_{1}^{2}+x_{2}^{2}\right)\right]$. The likelihood function for this process is given by

$$
\begin{aligned}
\frac{d P_{\theta, t}}{d \mu_{t}}= & \theta_{1} \exp \left\{-\theta_{1}\left[X_{1}^{2}(0)+X_{2}^{2}(t)+\int_{0}^{t}\left(X_{1}(s) d X_{1}(s)+X_{2}(s) d X_{2}(s)\right)\right]\right. \\
& +\theta_{2} \int_{0}^{t}\left[X_{1}(s) d X_{2}(s)-X_{2}(s) d X_{1}(s)\right] \\
& \left.-\frac{1}{2}\left(\theta_{1}^{2}+\theta_{2}^{2}\right) \int_{0}^{t}\left[X_{1}^{2}(s)+X_{2}^{2}(s)\right] d s\right\}
\end{aligned}
$$

(see Magiera and Stefanov (1989)). Thus the process belongs to the family defined by (1) with

$$
\begin{aligned}
Z_{1}(t) & =-X_{1}^{2}(0)-X_{2}^{2}(t)-\int_{0}^{t}\left(X_{1}(s) d X_{1}(s)+X_{2}(s) d X_{2}(s)\right), \\
Z_{2}(t) & =\int_{0}^{t}\left[X_{1}(s) d X_{2}(s)-X_{2}(s) d X_{1}(s)\right], \\
S(t) & =\int_{0}^{t}\left[X_{1}^{2}(s)+X_{2}^{2}(s)\right] d s, \\
\Phi(\theta) & =\frac{1}{2}\left(\theta_{1}^{2}+\theta_{2}^{2}\right) \text { and } \Psi(\theta)=-\log \theta_{1} .
\end{aligned}
$$


By Ito's formula, $Z_{1}(t)$ can be expressed by

$$
Z_{1}(t)=-\frac{1}{2}\left[X_{1}^{2}(0)+X_{2}^{2}(0)+X_{1}^{2}(t)+X_{2}^{2}(t)-2 t\right] .
$$

The model for processes defined by the system of stochastic differential equations (7) has often appeared in the literature devoted to problems of efficient and maximum likelihood estimation (Arato (1978), Taraskin (1974), Basawa and Prakasa Rao (1980, Chapter 9, Section 5), Magiera and Stefanov (1989)).

The conjugate prior density of the parameter $\theta=\left(\theta_{1}, \theta_{2}\right)$ takes the form

$f\left(\theta ; n_{0}, z_{1}, z_{2}, s\right)=K\left(n_{0}, z_{1}, z_{2}, s\right) \theta_{1}^{n_{0}} \exp \left[n_{0}\left(z_{1} \theta_{1}+z_{2} \theta_{2}-\frac{1}{2} s\left(\theta_{1}^{2}+\theta_{2}^{2}\right)\right)\right]$, $n_{0}>0,\left(z_{1}, z_{2}, s\right) \in \mathcal{Y}=(-\infty, \infty)^{2} \times(0, \infty)$. The norming constant is determined by

$$
\begin{aligned}
{[K} & \left.\left(n_{0}, z_{1}, z_{2}, s\right)\right]^{-1} \\
= & \int_{0}^{\infty} \int_{-\infty}^{\infty} \theta_{1}^{n_{0}} \exp \left[n_{0}\left(z_{1} \theta_{1}+z_{2} \theta_{2}-\frac{1}{2} s\left(\theta_{1}^{2}+\theta_{2}^{2}\right)\right)\right] d \theta_{1} d \theta_{2} \\
= & \int_{0}^{\infty} \theta_{1}^{n_{0}} \exp \left[n_{0}\left(z_{1} \theta_{1}-\frac{1}{2} s \theta_{1}^{2}\right)\right] d \theta_{1} \int_{-\infty}^{\infty} \exp \left[n_{0}\left(z_{2} \theta_{2}-\frac{1}{2} s \theta_{2}^{2}\right)\right] d \theta_{2} \\
= & \left(n_{0} s\right)^{-\left(n_{0}+1\right) / 2} \Gamma\left(n_{0}+1\right) \exp \left(\frac{n_{0} z_{1}^{2}}{4 s}\right) \mathcal{D}_{-n_{0}-1}\left(-\left(\frac{n_{0}}{s}\right)^{1 / 2} z_{1}\right) \\
& \times\left(\frac{2 \pi}{n_{0} s}\right)^{1 / 2} \exp \left(\frac{n_{0} z_{2}^{2}}{2 s}\right) \\
= & (2 \pi)^{1 / 2}\left(n_{0} s\right)^{-\left(n_{0}+3\right) / 2} \Gamma\left(n_{0}+1\right) \exp \left[\frac{n_{0}}{4 s}\left(z_{1}^{2}+2 z_{2}^{2}\right)\right] \\
& \times \mathcal{D}_{-n_{0}-1}\left(-\left(\frac{n_{0}}{s}\right)^{1 / 2} z_{1}\right) .
\end{aligned}
$$

In particular, for $z_{1}=0$ the prior distribution has the norming constant determined by

$$
\left[K\left(n_{0}, 0, z_{2}, s\right)\right]^{-1}=2^{n_{0} / 2} \pi^{1 / 2}\left(n_{0} s\right)^{-\left(n_{0}+2\right) / 2} \Gamma\left(\frac{n_{0}+1}{2}\right) \exp \left(\frac{n_{0} z_{2}^{2}}{2 s}\right) .
$$

According to Theorem 3(ii), $E\left(s \theta_{1}-1 / \theta_{1}\right)=z_{1}$ and $E \theta_{2}=z_{2} / s$.

In this case, the assumptions of the Lemma are satisfied for all $n_{0}>1$ and $(z, s) \in \mathcal{Y}$. Thus, (5) yields the following formulae:

$$
D\left(s \theta_{1}-\theta_{1}^{-1}\right)=E\left(z_{1}-s \theta_{1}+\theta_{1}^{-1}\right)^{2}=\frac{1}{n_{0}}\left(s+E \theta_{1}^{-2}\right),
$$




$$
\begin{gathered}
E\left(z_{1}-s \theta_{1}+\theta_{1}^{-1}\right)\left(z_{2}-s \theta_{2}\right)=0, \\
D\left(s \theta_{2}\right)=E\left(z_{2}-s \theta_{2}\right)^{2}=s / n_{0},
\end{gathered}
$$

which are valid for all $n_{0}>1$ and $(z, s) \in \mathcal{Y}$.

ExAmple 3 . Let $X(t), t \geq 0$, be a Poisson process with a parameter $\lambda$, starting at a fixed moment $t_{0}$. The likelihood function is given as follows:

$$
\frac{d P_{\lambda, t}}{d \mu_{t}}=\exp \left[X\left(t+t_{0}\right) \log \lambda-\lambda t-\lambda t_{0}\right] .
$$

This process satisfies (1) with $Z(t)=X\left(t+t_{0}\right), S(t) \equiv t, \theta=\log \lambda$, $\Phi(\theta)=\exp \theta$ and $\Psi(\theta)=t_{0} \exp \theta$.

The conjugate prior $\pi\left(\theta ; n_{0}, z, s\right)$ of the parameter $\theta$ has the density

$$
f\left(\theta ; n_{0}, z, s\right)=K\left(n_{0}, z, s\right) \exp \left[n_{0}\left(z \theta-\left(s+t_{0}\right) e^{\theta}\right)\right],
$$

$n_{0}>0,(z, s) \in \mathcal{Y}=(0, \infty)^{2}$, with

$$
K\left(n_{0}, z, s\right)=\frac{\left[n_{0}\left(s+t_{0}\right)\right]^{n_{0} z}}{\Gamma\left(n_{0} z\right)} .
$$

By Theorem 3(ii), $E(\exp \theta)=z /\left(s+t_{0}\right)$.

The conditions of the Lemma are satisfied for every $n_{0}>0$ and $(z, s) \in$ $\mathcal{Y}$. Thus the Lemma implies

$$
E\left[z-\left(s+t_{0}\right) \exp \theta\right]^{2}=\left(s+t_{0}\right) n_{0}^{-1} E(\exp \theta)=z / n_{0} .
$$

Hence, $D(\exp \theta)=z / n_{0}\left(s+t_{0}\right)^{2}$.

\section{References}

M. Arato (1978), On the statistical examination of continuous state Markov processes III, Selected Transl. in Math. Statist. and Probab. 14, 253-267.

O. E. Barndorff-Nielsen (1980), Conditionality resolutions, Biometrika 67, 293-310.

I. V. Basawa and B. L. S. Prakasa Rao (1980), Statistical Inference for Stochastic Processes, Academic Press, New York.

P. Diaconis and D. Ylvisaker (1979), Conjugate priors for exponential families, Ann. Statist. 7, 269-281.

R. Döhler (1981), Dominierbarkeit und Suffizienz in der Sequentialanalyse, Math. Operationsforsch. Statist. Ser. Statist. 12, 101-134.

I. S. Gradshteı̆n and I. M. Ryzhik (1971), Tables of Integrals, Sums, Series and Products, Nauka, Moscow (in Russian).

R. S. Liptser and A. N. Shiryaev (1978), Statistics of Random Processes, Vol. 2, Springer, Berlin.

R. Magiera and V. T. Stefanov (1989), Sequential estimation in exponential-type processes under random initial conditions, Sequential Anal. 8 (2), 147-167.

R. Magiera and M. Wilczyński (1991), Conjugate priors for exponential-type processes, Statist. Probab. Lett. 12, 379-384. 
A. F. Taraskin (1974), On the asymptotic normality of vector-valued stochastic integrals and estimates of drift parameters of a multidimensional diffusion process, Theory Probab. Math. Statist. 2, 209-224.

RYSZARD MAGIERA

INSTITUTE OF MATHEMATICS

TECHNICAL UNIVERSITY OF WROCłAW

WYBRZEŻE WYSPIAŃSKIEGO 27

50-370 WROCłAW, POLAND

Received on 15.4.1993;

revised version on 25.5.1994 